\title{
Pair interaction and phase separation in mixtures of colloids and excluded volume polymers
}

\author{
R. Tuinier, ${ }^{* a}$ D. G. A. L. Aarts, ${ }^{b}$ H. H. Wensink ${ }^{b}$ and H. N. W. Lekkerkerker ${ }^{b}$ \\ ${ }^{a}$ Forschungszentrum Jülich, Institut für Festkörperforschung, 52425, Jülich, Germany. \\ E-mail: r.tuinier@fz-juelich.de. \\ ${ }^{b}$ Van't Hoff Laboratory, Debye Research Institute, University of Utrecht, Padualaan 8, \\ 3584, CH Utrecht, The Netherlands
}

Received 13th May 2003, Accepted 3rd July 2003

First published as an Advance Article on the web 4th August 2003

The interactions in polymer-colloid mixtures and their phase stability are calculated using the adsorption method. Starting from appropriate expressions for the correlation length and the osmotic pressure of a polymer solution in the excluded volume interaction limit, this method allows computation of the potential between two plates, two spheres and between a sphere and a plate. The results are in close agreement with computer simulation results. Extended to a many body system of colloids and polymers, the same approach allows one to calculate the thermodynamic properties and phase behavior. Results are presented for the phase behavior of polymer-sphere and polymer-platelet mixtures. The agreement with experimental data is satisfactory and helps to better explain the polymer-colloid phase behavior.

\section{Introduction}

Adding non-adsorbing polymers to colloidal dispersions leads to an effective attraction between the colloids. The attraction originates from the less accessible regions for the polymer chains near the colloid surfaces. ${ }^{1}$ When the concentration of both components exceeds a certain level it leads to a phase separation. Colloidal gas-liquid, gas-solid and a three phase gas-liquid-solid coexistence are found when polymers are added to a mixture of colloidal spheres. ${ }^{2}$ The phase behavior becomes different in case of anisometric colloidal particles due to the possibility of liquid crystal formation. In mixtures of colloidal rods and polymer, Buitenhuis et al ${ }^{3}$ could distinguish between two isotropic (I) phases (a dilute and a concentrated one), and a nematic liquid crystal $(\mathrm{N})$ phase. Both biphasic and triphasic equilibria were observed. Van der Kooij et al. ${ }^{4}$ investigated the phase behavior of platelet-polymer mixtures and found two isotropic phases, a nematic and a columnar liquid crystal (C) phase. These experimental studies ${ }^{2-4}$ were performed on colloidal particles with well-defined shapes mixed with synthetic polymer. It should be stressed that both under natural and practical conditions dispersed colloidal particles are often in the presence of polymers. For instance, polymers and colloids are joined together in food as polysaccharides and proteins. ${ }^{5}$ Under physiological conditions concentrated DNA, sometimes modeled as a rod, is mixed with polysaccharides and/or proteins ${ }^{6}$ and platelets mixed with polysaccharides can be found in the soil. ${ }^{7}$

Theoretical work on polymer-colloid mixtures ${ }^{8-17}$ has mainly focused on simplifying the polymers by treating them as penetrable hard spheres (the so-called AO model ${ }^{8-11}$ ) with a radius equal to the polymer's radius of gyration. In some cases this description is adequate, but as soon as the relevant polymer concentrations become larger than the overlap concentration it fails; it turns out that the predicted phase transitions occur at too small polymer concentrations. Several approaches have recently been developed that aim at a better description of the polymer chains. These include polymer reference interaction site models (denoted as PRISM), ${ }^{18-20}$ density-functional theory ${ }^{21,22}$ a Gaussian core model, ${ }^{23-30}$ and field theories. ${ }^{31,32}$ In order to attain a proper prediction of the depletion potential and the resulting phase behavior, it is however convenient to keep the descriptions simple and thereby insightful, while not losing essential physics. We are therefore inspired by the original work of Asakura and Oosawa $^{8}$ and Vrij. ${ }^{9}$ In a number of recent papers ${ }^{33-35}$ we have extended their ideas and included polymeric excluded volume interactions. It is of great importance to put the proper depletion layer thickness into a statistical mechanical framework. Instead of taking the polymer's radius of gyration we calculate the depletion layer thickness from the negative adsorption around a colloidal particle. ${ }^{34}$ This ensures, for instance, that the depletion layer thickness depends properly on the polymer-colloid size ratio; at large size ratios the polymer chains can make more configurations near the colloid and the relative curvature for the chain determines the range over which it is excluded. Inserting the excluded volume interactions between the polymer segments is made possible by incorporating results from renormalization group ( $\mathrm{RG}$ ) theory for the osmotic pressure and correlation length of interacting polymers (polymers with excluded volume interaction). ${ }^{36}$ When excluded volume interactions between the segments are incorporated the depletion thickness becomes concentration-dependent, as already pointed out by Joanny et $a l .{ }^{37}$ using a scaling analysis.

In this paper we present a general scheme on how curvature effects and segment excluded volume interactions can be incorporated in a theory for the interaction between colloidal particles and for the phase behavior of polymer-colloid mixtures. It is shown that using the appropriate depletion layer thickness enables a calculation of the depletion interaction between colloidal particles immersed in a solution of interacting polymers. The results are in good agreement with computer simulations. Calculations on the phase behavior of sphere-polymer and plate-polymer mixtures demonstrate how polymer excluded volume and curvature effects influence the phase behavior of colloid-polymer mixtures. The results are compared with experiments on polymer-sphere and polymerplatelet systems. 


\section{Depletion interaction from the adsorption method}

In this section, the interaction potentials between colloidal particles are considered for three geometry types: plate-plate, sphere-sphere, and sphere-plate. As a tool to calculate these interactions we use the adsorption method, which follows from the generalized Gibbs adsorption equation. ${ }^{38}$ It leads to the following expression for the interaction potential $W(h)$ (see, for instance, the appendix in Mao et al. ${ }^{39}$ ):

$$
W(h)=-\int_{-\infty}^{\mu} \mathrm{d} \mu^{\prime}[\Gamma(h)-\Gamma(\infty)]
$$

where $\mu$ is the chemical potential of the polymers in solution and $\Gamma(h)$ is the adsorbed amount of polymer segments or the excess polymer segment concentration when the colloidal particles are a distance $h$ apart. Note that the interaction potential obtained from eqn. (1) is the effective potential between two colloidal particles; the degrees of freedom of the polymers are integrated out. It is convenient to rewrite eqn. (1) using the Gibbs-Duhem relation, $n_{\mathrm{p}}{ }^{-1} \mathrm{~d} \Pi=\mathrm{d} \mu:^{33}$

$$
W(h)=-\int_{0}^{n_{\mathrm{p}}} \mathrm{d} n_{\mathrm{p}}^{\prime} \frac{1}{n_{\mathrm{p}}^{\prime}}\left(\frac{\partial \Pi}{\partial n_{\mathrm{p}}^{\prime}}\right)[\Gamma(h)-\Gamma(\infty)],
$$

in terms of the polymer bulk concentration, $n_{\mathrm{p}}$, which is equal to $3 \phi_{\mathrm{p}} /\left(4 \pi R_{\mathrm{g}}^{3}\right)$ with $\phi_{\mathrm{p}}$ the relative polymer concentration (三1 at the overlap concentration), and the osmotic pressure $\Pi$ of the polymer solution. From RG theory it follows that the osmotic compressibility can be written as a function of the relative bulk polymer concentration $\phi_{\mathrm{p}}$ as: ${ }^{36}$

$$
\frac{\partial(\beta \Pi)}{\partial n_{\mathrm{p}}}=1+2.63 \phi_{\mathrm{p}}\left(\frac{1+3.25 \phi_{\mathrm{p}}+4.15\left(\phi_{\mathrm{p}}\right)^{2}}{1+1.48 \phi_{\mathrm{p}}}\right)^{0.309}
$$

here $\beta=1 / k T$. The adsorbed amount $\Gamma(h)$ can be written as a volume integral over the polymer (segment) density profile $n(\boldsymbol{r})$ :

$$
\Gamma(h)=n_{\mathrm{p}} \int_{V} \mathrm{~d} \boldsymbol{r}\left(\frac{n(\boldsymbol{r})}{n_{\mathrm{s}}}-1\right)
$$

with $n_{\mathrm{s}}$ the bulk segment concentration, related to $n_{\mathrm{p}}$ by $n_{\mathrm{s}} \equiv n_{\mathrm{p}} M$, with $M$ being the number of polymer segments in a polymer chain. In eqn. (4) the volume $V$ refers to the volume available to the polymer chains.

\subsection{Depletion layer near a flat plate and around a sphere}

Let us map the actual polymer density profiles onto a stepfunction profile involving the correct depletion layer thickness, i.e. we replace the smooth density profile by a Heaviside (step-) function, with thickness $\Delta$, to take the depletion effect into account. The correct depletion thickness follows from imposing the condition that the negative adsorption around a single colloidal particle must be the same for the smooth polymer density profile and the step-function profile. The required quantity for computing the adsorbed amount $\Gamma(h)$ is the depletion layer thickness around a sphere in a polymer solution with excluded volume segment interaction. From the sphere result, the flat plate result can be derived straightforwardly.

For spherical particles we use the fact that the negative adsorption around a particle is captured in the free energy $F_{1}$ of immersing a macroscopic particle into a dilute polymer solution. ${ }^{26}$ In case of a step-function with depletion thickness, $\triangle$, it becomes:

$$
\beta F_{1}=\frac{4 \pi}{3} n_{\mathrm{p}}(R+\Delta)^{3}=-\Gamma_{\text {single }}+\frac{4 \pi}{3} n_{\mathrm{p}} R^{3},
$$

where $\Gamma_{\text {single }}$ is the adsorbed amount around a single sphere.
Here, we follow two approaches to derive the depletion layer thickness. In the first approach the free energy of immersing a sphere into a polymer solution with excluded volume interaction is calculated and from eqn. (5) the depletion thickness will be obtained subsequently. In the low-density limit of interacting polymers, Hanke et al. ${ }^{31}$ calculated the flat and curvature-dependent terms of the surface tension, $\sigma$ :

$$
\beta \sigma=n_{\mathrm{p}}\left[z_{1} R_{\mathrm{g}}+q z_{2} R_{\mathrm{g}}+q^{2} z_{3} R_{\mathrm{g}}+\ldots\right],
$$

where $q=R_{\mathrm{g}} / R$ and with $z_{1} \approx 1.071, z_{2} \approx 0.869$, and $z_{3} \approx$ $-0.0399 .{ }^{31,34}$ The quantity $\sigma$ is directly related with the free energy of immersion in the dilute limit via:

$$
\beta F_{1}=n_{\mathrm{p}} \frac{4 \pi}{3} R^{3}+4 \pi R^{2} \beta \sigma
$$

leading to:

$$
\beta F_{1}=\frac{4 \pi}{3} n_{\mathrm{p}} R^{3}\left(1+3 z_{1} q+3 z_{2} q^{2}+3 z_{3} q^{3}+\ldots\right) .
$$

Neglecting higher order terms and equating eqns. (8) and (5), the following expression for the depletion layer thickness $\Delta$ up to third order in $R_{\mathrm{g}} / R$ can be found: ${ }^{34}$ :

$$
\frac{\Delta}{R}=\left[1+3 z_{1} q+3 z_{2} q^{2}+3 z_{3} q^{3}\right]^{\frac{1}{3}}-1 .
$$

In order to obtain the polymer concentration dependence of the depletion layer thickness, we assume this expression holds but with the bulk correlation length $\xi_{\mathrm{b}}$ replacing the polymer radius of gyration, $R_{\mathrm{g}}:{ }^{34}$

$$
\frac{\Delta}{R}=\left[1+3.213 \frac{\xi_{\mathrm{b}}}{R}+2.607\left(\frac{\xi_{\mathrm{b}}}{R}\right)^{2}-0.120\left(\frac{\xi_{\mathrm{b}}}{R}\right)^{3}\right]^{\frac{1}{3}}-1
$$

The correlation length $\xi_{\mathrm{b}}$ is taken from renormalization group theory (ref. 36; see also 34). The depletion layer thickness near a flat plate follows from the leading order term of the expansion in $1 / R$ :

$$
\Delta=1.071 \xi_{\mathrm{b}}
$$

A second approach we took here is using the polymer density profile around a sphere in a polymer solution with excluded volume interaction to calculate the negative adsorption using:

$$
\Gamma_{\text {single }}=4 \pi n_{\mathrm{p}} \int_{R}^{\infty} \mathrm{d} r r^{2}\left(\frac{n(r)}{n_{\mathrm{s}}}-1\right)
$$

The depletion layer thickness can now be obtained from inserting eqn. (12) into eqn. (5). There is no exact analytical solution of the polymer density available for the Edwards-de Gennes equation that needs to be solved (see ref. 40), except in specific limits. An accurate expression was recently obtained for the polymer density in a semi-dilute polymer solution around a sphere: ${ }^{40}$

$$
\begin{aligned}
\frac{n(x)}{n_{\mathrm{s}}}= & \left(\frac{x}{R+x}\right)^{2}\left\{1+\frac{R}{x}\left(\frac{2}{3} \tanh \left(\frac{x}{\xi_{\mathrm{w}}}\right)\right.\right. \\
& \left.\left.+\frac{4}{3}\left[1-\exp \left(-\frac{2 x}{\xi_{\mathrm{w}}}\right)\right]\right)+\left(\frac{R}{x}\right)^{2} \tanh ^{2}\left(\frac{x}{\xi_{\mathrm{w}}}\right)\right\},
\end{aligned}
$$

with $\xi_{\mathrm{w}}$ being the relevant polymer depletion length scale near a wall. Here, $x$ is defined as $r-R$, where $r$ is the distance from the center of the sphere. Inserting this equation into eqn. (12) and using eqn. (5) leads to the expression:

$$
\begin{aligned}
\frac{\Delta}{R} & =\left[1+3 \frac{\xi_{\mathrm{w}}}{R}+\left(1+\frac{\pi^{2}}{12}\right)\left(\frac{\xi_{\mathrm{w}}}{R}\right)^{2}\right]^{\frac{1}{3}}-1 \\
& =\left[1+3.213 \frac{\xi_{\mathrm{b}}}{R}+2.09\left(\frac{\xi_{\mathrm{b}}}{R}\right)^{2}\right]^{\frac{1}{3}}-1 .
\end{aligned}
$$


For consistency, we take the right concentration dependence of the correlation length by setting $\xi_{\mathrm{w}}=1.071 \xi_{\mathrm{b}}$, with the correlation length again $\xi_{\mathrm{b}}$ taken from the RG theory. ${ }^{36}$ The two independent expressions (10) and (14) predict very comparable size ratio dependences up to size ratio unity. The disadvantage of eqn. (10) is that higher order terms are unknown, so it is not accurate for large curvature and it strictly only applies for the dilute case. Eqn. (14) was derived explicitly for the semi-dilute regime and covers the entire size ratio range but is a mean-field result (although we insert the right scaling exponents via the $\xi_{\mathrm{b}}$ from the RG theory) and was not derived for the dilute case.

\subsection{Interaction between colloidal particles mediated by interacting polymers}

Two plates. We start with the simple situation of two parallel flat plates immersed in a polymer solution. This is the simplest example to test our theory in absence of curvature effects. The excess adsorbed amount of polymer segments between two flat plates, using the depletion layer concept, follows as the overlap volume times the polymer concentration $n_{\mathrm{p}}$ (the parallel plate version of eqn. (5) in ref. 34).

$$
\Gamma(h)-\Gamma(\infty)=\left\{\begin{array}{ll}
n_{\mathrm{p}}(2 \Delta-h) & \text { for } h \leq 2 \Delta \\
0 & \text { for } h>2 \Delta
\end{array},\right.
$$

For two plates immersed in a solution of interacting polymers we have according to eqn. (11): $\Delta=1.071 \xi_{\mathrm{b}}$. The interaction potential can be calculated using eqn. (2). Louis et $a .^{27}$ suggested a simpler form of the interaction potential, assuming that the potential is linear in $h$ under all conditions. In order to use their analytical result they use results from computer simulations. Here, we use independent theoretical results and insert them into the generalized Gibbs adsorption equation. Computer simulations of self-avoiding random walks (SAW) in a 'background' solvent between two parallel flat plates have been done by Bolhuis et al. ${ }^{24}$ and yield the interaction potential. In Fig. 1, the interaction potentials for three polymer concentrations are plotted as a function of the relative (parallel) plate separation distance, $h / R_{\mathrm{g}}$. The values of the contact potentials of the SAW simulations (symbols) correspond reasonably well to our theoretical predictions (full curves). The contact potential is only perceptibly smaller and the initial slope of the potential is somewhat less steep when

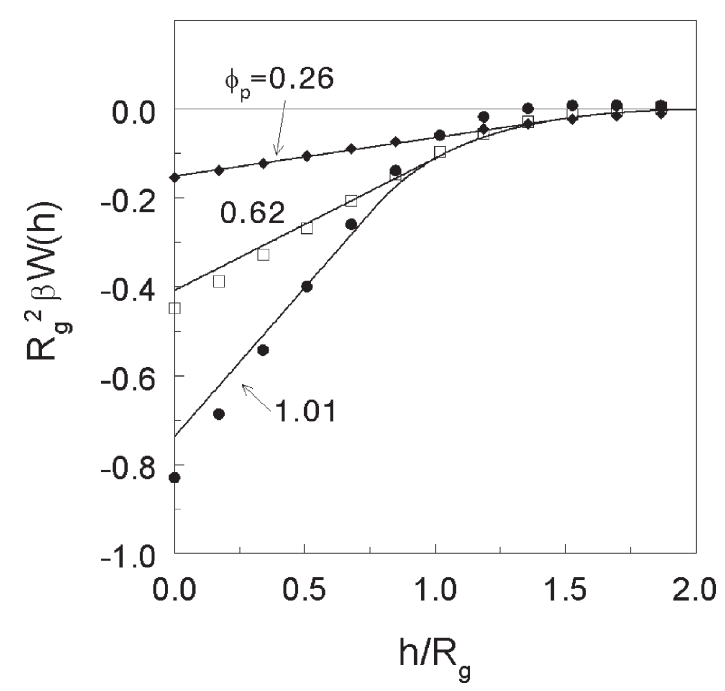

Fig. 1 Interaction potential between two hard plates immersed in a solution with self-avoiding walk polymers as a function of the distance between the plates for three polymer concentrations as indicated. The symbols refer to SAW simulation results from references 23 and 24 and the full curves are the prediction of the proposed theory for interacting polymers (polymer concentrations are indicated in the plot). comparing our results with the computer simulations. These differences can be explained by a small overestimation of the polymer osmotic pressure in the SAW simulations as compared to the RG theory (see Fig. 11 in ref. 24). This may be related to the fact that the SAW simulations were made with chains of $N=100$ segments whereas the theory in the excluded volume limit applies strictly for $N \rightarrow \infty$. When we replace the osmotic compressibility from the RG theory [eqn. (3)] with the compressibility from the SAW simulation results, our theory is even in closer agreement with the simulation results. At any rate, the range and value of the interaction potentials agree reasonably well.

Two spheres. For two colloidal hard spheres in a polymer solution, the adsorption follows from eqn. (4) (see also eqn. (5) in ref. 34) as:

$$
\begin{aligned}
& \Gamma(h)-\Gamma(\infty) \\
& \quad= \begin{cases}\frac{2}{3} \pi n_{\mathrm{p}} \Delta^{3}\left(1-\frac{h}{2 \Delta}\right)^{2}\left(2+\frac{3 R}{\Delta}+\frac{h}{2 \Delta}\right) & \text { for } h \leq 2 \Delta \\
0 & \text { for } h>2 \Delta\end{cases}
\end{aligned}
$$

(see also $\mathrm{Vrij}^{9,10}$ ). Inserting this expression into eqn. (2) and using the size ratio- and concentration-dependent depletion layer thickness [either eqns. (10) or (14)] together with the osmotic compressibility from the RG theory [eqn. (3)], gives the interaction potential. Previously, we have shown that our concept correctly describes the universal de Gennes scaling law for the contact potential of spherical colloidal particles in a semi-dilute polymer solution. ${ }^{34}$ Here, we compare our theoretical prediction with SAW simulation results. ${ }^{27}$ Louis et $a .^{27}$ made grand-canonical computer simulations on two hard spheres in a box with self-avoiding walk (SAW) polymer chains with a chain length of 500 segments. The depletion force on the spheres could be derived from these simulation results and they are redrawn in Fig. 2(a-c) for three size ratios and various polymer concentrations, as indicated. The force $f(h)$ is easily calculated from the interaction potential [eqn. 17 plus 2 yield an expression for $f(h)$ ] using:

$$
f(h)=-\frac{\partial W(h)}{\partial h} .
$$

The full curves in Fig. 2(a-c) are the predictions from our theory using eqn. (10) (full curves) and (14) (dashed curves) for the depletion layer thickness around a sphere. The overall agreement is excellent for all size ratios, taking into account the significant fluctuations (see ref. 41) in the depletion force. For $q=1.68$ we expect eqn. (10) to become less accurate since the higher order curvature terms are unknown. For this size ratio it indeed seems that eqn. (14) (dashed curves) for the depletion layer thickness, which is valid for arbitrary size ratio, is slightly better.

Results for the contact potential are given in Fig. 3. The results from the present theory (using eqn. (10) for $\Delta$ ) again agree reasonably with the simulation data (which are the integrated results for the force). The deviation is small but increases with polymer concentration. This may be due to a difference in the osmotic pressure used, as discussed in the previous section. Using the accurate solution of the Edwards-de Gennes differential eqn. (14) very similar results are obtained (dashed curves), the attraction being only very slightly weaker.

Recently, self-avoiding walk simulations were performed by Bolhuis et $a l^{42}$ to compute the effective pair interaction between two relatively small spheres, with a radius equal to 5 segment lengths in a reservoir of interacting long polymer chains (2000 segments). For $q=R_{\mathrm{g}} / R=7.78$ their results are redrawn for four polymer concentrations in Fig. 4(a). In Fig. 4(b) the results of our analytical theory are given using the Edwards-de Gennes expression [eqn. (14)] for the 

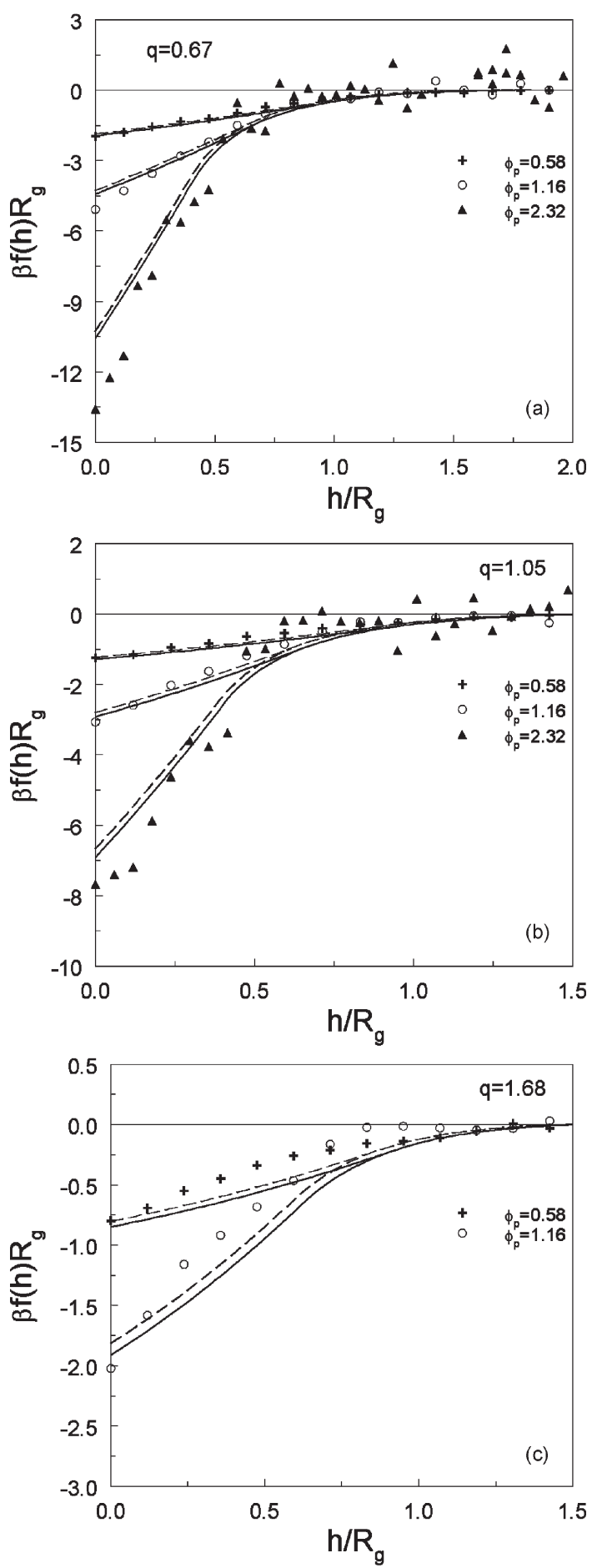

Fig. 2 The force between two spheres immersed in a solution with self-avoiding walk polymers as a function of the distance between the spheres for $R_{\mathrm{g}} / R=q=0.67$ (a), $q=1.05$ (b) and $q=1.68$ (c) for polymer concentrations of $\phi_{\mathrm{p}}=0.58,1.16$, and 2.32, as indicated. The symbols refer to SAW simulation results redrawn from ref. 27. Curves represent the predictions of our analytical theory for polymers in the excluded volume limit by using eqn. (10) (full) or eqn. (14) (dashed) for the depletion thickness around the spheres.

depletion thickness. The theoretical results are in qualitative agreement with the simulations. This demonstrates that, using the solution of the Edwards-de Gennes diffusion equation, ${ }^{40}$ our theory is also applicable for large and intermediate polymer-colloid size ratios. Quantitatively, the theoretical data are about a factor of 1.3 larger than the simulation results. There are three main reasons for this deviation. Firstly, eqn. (14) is a mean-field result although the right scaling behavior for the depletion layer thickness is inserted using RG results.

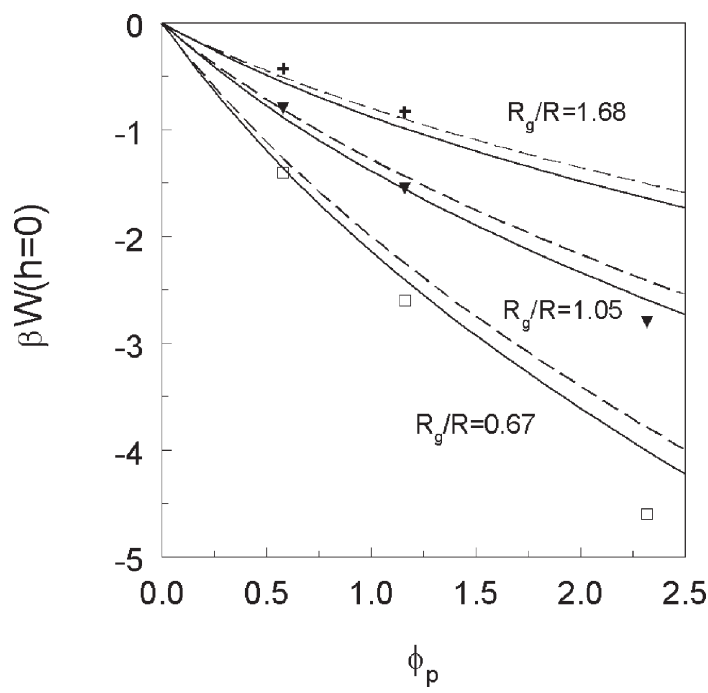

Fig. 3 Contact potential between two spheres immersed in a solution with self-avoiding walk polymers as a function of polymer concentration. The symbols refer to SAW simulation results, redrawn from ref. 27 and the curves are the prediction of the proposed theory for excluded volume polymers $\left(R_{\mathrm{g}} / R\right.$ values are indicated in the plot). The depletion layer thickness was computed from eqn. (10) (full curves) or eqn. (14) (dashed curves).
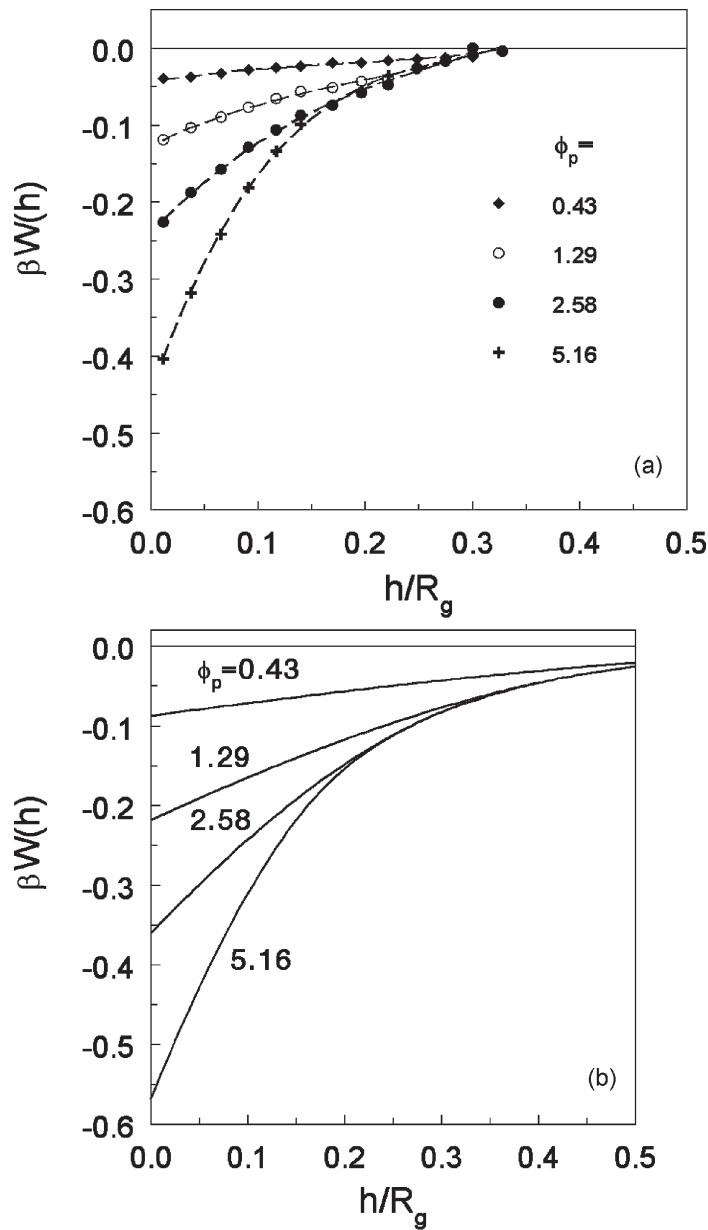

Fig. 4 (a) Computer simulation results for the interaction potential between two spheres immersed in a solution with self-avoiding walk polymers as a function of the interparticle distance $h$ for $q=7.78$. Data points were redrawn from ref. 42. Dashed curves are a guide to the eye. (b) Interaction potential between two small spheres immersed in a solution with self-avoiding walk polymers as in Fig. 4(a) using the adsorption method as a function of the interparticle distance $h$. 
Secondly, our theory assumes that the polymer chains are infinitely flexible. The simulations were performed with colloidal spheres that have a radius equal to only 5 segment lengths. This means that the polymer chains are not fully Gaussian on the sphere length scale, which weakens the effective interaction. ${ }^{43}$ Thirdly, in our theory we use results from the RG theory, which assumes that the chains have a full excluded volume interaction. Around a small sphere only several segments interact which leads to an effective correlation length close to the sphere (that determines the depletion thickness) that deviates from the full RG bulk correlation length. Still, the agreement is very good when realizing that the theory presented does not contain adjustable parameters and is completely independent of the simulations. The theory of Louis et al. ${ }^{27}$ that employs the results from the SAW simulations, predicts a more accurate contact potential, but misses the tail of the long-range attraction (see Fig. 4 in ref. 42).

Very recently, the depletion potential between two spheres in a polymer solution has been measured using the scanning optical tweezer technique. ${ }^{44}$ This enables a test of theories and computer simulations for the pair potential as a function of size ratio and polymer density. By trapping two silica spheres with a diameter of $1.25 \mu \mathrm{m}$, Verma et al. ${ }^{44}$ measured the interaction between them while immersed in a solution of DNA molecules with a radius of gyration close to $500 \mathrm{~nm}$ : the size ratio $R / R_{\mathrm{g}}$ is 1.25 . The solvent had an ionic strength of 10 $\mathrm{mM}$ (Debye length $\approx 3 \mathrm{~nm}$ ). In Fig. 5 their results are reproduced for concentrations of 140,190 , and $280 \mu \mathrm{g} \mathrm{mL}^{-1}$ (symbols). The DNA concentrations now have to be matched to the relative polymer concentration $\phi p$. If $3 \mathrm{M} /\left(N_{\mathrm{av}} 4 \pi R_{\mathrm{g}}^{3}\right)$ is taken as the overlap concentration in $\mathrm{g} \mathrm{L}^{-1}$, one arrives at $96 \mu \mathrm{g}$ $\mathrm{mL}^{-1}$. Hence 140,190 , and $280 \mu \mathrm{g} \mathrm{mL}^{-1}$ correspond to $\phi_{\mathrm{p}}=1.46,1.98$ and 2.92. The theoretical results for these three concentrations for a colloid-to-polymer size ratio $R / R_{\mathrm{g}}=1.25$ are plotted in Fig. 5 as well (full curves) using eqn. (10) for the depletion thickness. It is remarkable how our simple theory gives a fair description of the experimental data. The magnitude of the experimentally determined contact potentials is well described with the present theoretical results. It is noted that the experimental data cross whereas our approach predicts collapsing of the long-ranged potential values. Deviation details may be due to the fact that DNA is a polyelectrolyte and not a neutral polymer with excluded volume interaction.

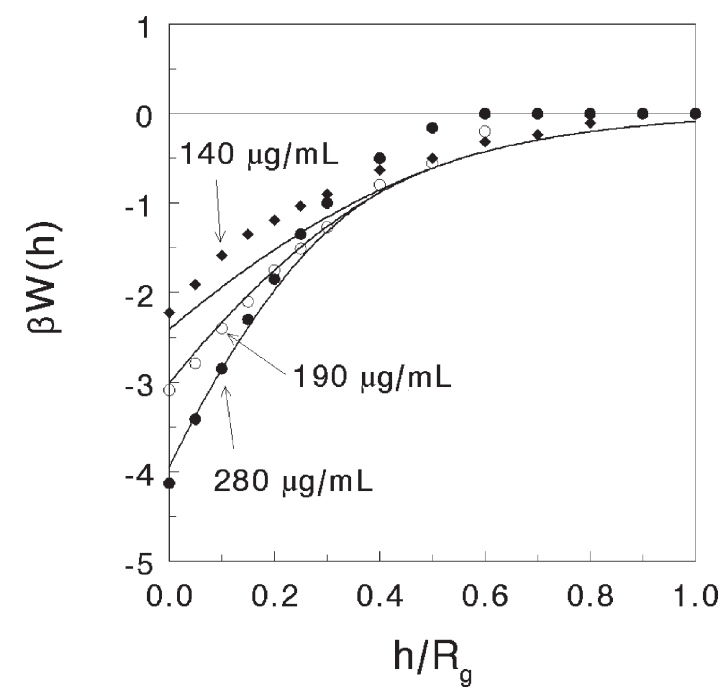

Fig. 5 Comparison of the interaction potential with experimental data. Measured interaction potentials between two silica spheres, with a diameter of $1.25 \mu \mathrm{m}$ in a non-adsorbing solution with DNA molecules with a radius of gyration of $500 \mathrm{~nm}$ of Verma et al. ${ }^{44}$ are represented by the symbols. The results of the present theory are given by the full curves, and are given for three polymer concentrations, as indicated.
Further, it is realized that the RG theory is used in the excluded volume limit and that DNA does not fully obey this description. ${ }^{36,44}$ At least it is satisfying that the order of magnitude is corresponding for the interaction between two spheres in a concentrated polymer solution. In the future, a better test of the theory will be a measurement for the depletion potential in a solution with non-adsorbing long, flexible neutral polymer chains. At present, such data are not yet available.

Sphere-plate. In the last decade, several experimental techniques have been developed that allow a direct measurement of the force between a flat plate and a colloidal sphere. They include atomic force microscopy (AFM), ${ }^{45}$ total internal reflection microscopy (TIRM), ${ }^{46,47}$ and laser pressure radiation techniques. ${ }^{48}$ Our method in fact allows prediction of the force between a plate and a sphere due to non-adsorbing polymers with excluded volume interaction. In order to calculate the interaction between a flat plate and a sphere in a polymer solution we first derive an expression for the overlap volume of the depletion layers around the flat plate and the sphere:

$$
\begin{aligned}
& \frac{\Gamma(h)-\Gamma(\infty)}{n_{\mathrm{p}}} \\
& \quad= \begin{cases}\frac{4}{3} \pi\left(R+\Delta_{s}\right)^{3} & \text { for } h<\Delta_{\mathrm{w}}-2 R-\Delta_{\mathrm{s}} \\
\frac{1}{3} \pi\left(\Delta_{s}+\Delta_{\mathrm{w}}-h\right)^{2} & \left(3 R+2 \Delta_{s}-\Delta_{\mathrm{w}}+h\right) \\
0 & \text { for } \Delta_{\mathrm{w}}-2 R-\Delta_{\mathrm{s}} \leq h \leq \Delta_{\mathrm{w}}+\Delta_{\mathrm{s}} \\
0 & \text { for } h>\Delta_{\mathrm{w}}+\Delta_{\mathrm{s}}\end{cases}
\end{aligned}
$$

where $\Delta_{\mathrm{s}}$ is the depletion layer thickness around the sphere and $\Delta_{\mathrm{w}}$ is the depletion layer thickness near a single plate. The left-hand side of eqn. (18) equals the excess negative adsorption between a plate and a sphere separated by a distance $h$, with $h+R$ being the distance between the plate and the sphere center, divided by the polymer concentration. The spheres are usually large compared to the polymer coils in a TIRM experiment and the expression for the negative adsorption is restricted to the last two lines $\left(R>\Delta_{\mathrm{w}}\right)$. The interaction now follows from insertion of eqn. (18) into eqn. (2) and using eqn. (3) for the osmotic compressibility.

\section{Phase behavior of mixtures of colloids and interacting polymers}

In this section, we incorporate the theoretical results obtained so far in a statistical mechanical framework to predict the phase behavior of mixtures of colloids and excluded volume polymers. As a starting tool we use the free volume treatment of the original AO model.

\subsection{Free volume theory}

The semi-grand canonical potential $\Omega\left(N, V, T, \mu_{\mathrm{p}}\right)$ which consists of a colloidal (reference) part, $F_{\mathrm{c}}(N, V, T)$ and a perturbation contribution due to the presence of the polymer chains, is formally written as: ${ }^{13}$

$$
\Omega\left(N, T, V, \mu_{\mathrm{p}}\right)=F_{\mathrm{c}}(N, T, V)-\int_{-\infty}^{\mu_{\mathrm{p}}} \mathrm{d} \mu_{\mathrm{p}}^{\prime} N_{\mathrm{p}} .
$$

This exact expression can be considered as the many-body analogue of the Gibbs-adsorption [eqn. (1)]. The solvent is considered as background. To make progress an expression for $N_{\mathrm{p}}$, the number of polymer chains in the system, as a 
function of the reservoir chemical potential of polymers, $\mu_{\mathrm{p}}$, is required. The following mean-field approximation is made: ${ }^{2,13}$

$$
N_{\mathrm{p}}=n_{\mathrm{p}}^{\mathrm{r}}\left\langle V_{\text {free }}\right\rangle_{0},
$$

where $n_{\mathrm{p}}^{\mathrm{r}}$ is the number of polymers per unit volume in a reservoir, in equilibrium with the system of polymers and colloids. The reservoir is separated from the system by a semi-permeable membrane; it allows passing through solvent and polymers but not colloidal particles. The quantity $\left\langle V_{\text {free }}\right\rangle_{0}$ is the available free volume for the polymer chains in the system. The index 0 indicates that the configurations of the colloidal particles are assumed to be unaffected by the addition of polymers. Defining the free volume fraction, $\alpha \equiv\left\langle V_{\text {free }}\right\rangle_{0} / V$, and using the Gibbs Duhem relation (see ref. 35) it follows in dimensionless notation:

$$
\omega=f_{\mathrm{c}}-\int_{0}^{\phi_{\mathrm{p}}^{\mathrm{r}}} \mathrm{d} \phi_{\mathrm{p}}^{\mathrm{r}_{1}}\left[\alpha \frac{\partial \tilde{\Pi}}{\partial \phi_{\mathrm{p}}^{\mathrm{r}_{1}}}\right] .
$$

where $\omega=\beta \Omega v_{\text {coll }} / V, f_{\mathrm{c}}=\beta F_{\mathrm{c}} v_{\mathrm{coll}} / V$ and $\tilde{\Pi}=\beta v_{\mathrm{coll}} \Pi\left(v_{\mathrm{coll}}\right.$ is the volume of the colloidal particle). The free volume fraction for the polymer chains, $\alpha$, can be obtained from scaled particle theory (SPT). ${ }^{49,50}$ The free volume fraction depends on the colloid concentration, the size of the depletion layers that surround the colloidal particles and the colloid size itself. For interacting polymer chains the depletion layers are concentration dependent (see section 2.1). Further, the osmotic compressibility becomes concentration-dependent.

\subsection{Spherical colloid dispersion with added excluded volume polymer}

For a mixture of hard spheres mixed with interacting polymers the free volume theory was recently extended by us. ${ }^{35}$ In case of spheres $v_{\text {coll }}$ equals $4 \pi R^{3} / 3$ and the free volume fraction reads:

$\alpha=\left(1-\phi_{\mathrm{s}}\right) \exp \left[-A\left(\frac{\phi_{\mathrm{s}}}{1-\phi_{\mathrm{s}}}\right)-B\left(\frac{\phi_{\mathrm{s}}}{1-\phi_{\mathrm{s}}}\right)^{2}-C\left(\frac{\phi_{\mathrm{s}}}{1-\phi_{\mathrm{s}}}\right)^{3}\right]$,

where $\phi_{\mathrm{s}}$ is the volume fraction of spheres and where the parameters $A, B$ and $C$ read:

$$
\begin{gathered}
A=3 \frac{\Delta}{R}+3\left(\frac{\Delta}{R}\right)^{2}+\left(\frac{\Delta}{R}\right)^{3} \\
B=\frac{9}{2}\left(\frac{\Delta}{R}\right)^{2}+3\left(\frac{\Delta}{R}\right)^{3}, \quad C=3\left(\frac{\Delta}{R}\right)^{3},
\end{gathered}
$$

The osmotic compressibility of the polymer solution is given by eqn. (3) for polymer chains in the excluded volume limit. In contrast to the ideal chain case the compressibility is now concentration-dependent. For $f_{\mathrm{c}}$ in eqn. (21) we use the expressions given in ref. 35 for the fluid and solid (fcc crystal) phases. The depletion layer thickness of excluded volume interacting polymer chains around spheres is the essential ingredient for the free volume fraction in eqn. (22). In ref. 35 we discussed the main findings of the theory. The most important difference between interacting and ideal polymer chains is that the binodal curves are shifted to higher polymer coil volume fractions for interacting polymers for larger values of the size ratio $q$. Using PRISM, Fuchs and Schweizer ${ }^{19}$ were the first to report that polymer-polymer interactions lead to an upward shift of the polymer concentration at the spinodal. The inclusion of the excluded volume interaction between the polymer segments in the free volume theory ${ }^{35}$ led to a significantly better quantitative prediction of the liquid-crystal and gas-liquidcrystal coexistences for the intensely investigated mixture of PMMA spheres mixed with polystyrene in cis-decalin with
$q=R_{\mathrm{g}} / R=0.57 .^{2,51}$ Moussaid et al. ${ }^{51}$ compared the triple coexistence liquids at $q=0.24,0.37$ and 0.57 . For $q=0.24$ and 0.37 the theory for ideal polymers mixed with hard spheres ${ }^{13}$ still gives a fair quantitative prediction of the concentrations of polymers and colloidal spheres in the colloidal liquid at the triple point. For $q=0.57$, however, the theory for ideal polymer chains ${ }^{13}$ predicts a colloid volume fraction of 0.499 and a polymer coil volume fraction of $0.00277,{ }^{51}$ while the experimental results gave a colloid volume fraction of 0.444 and a polymer coil volume fraction of $0.1,{ }^{50}$ so the theory with ideal polymer chains underestimates the polymer concentration by a factor of 36 . For hard spheres mixed with excluded volume polymer chains we find a colloid volume fraction of 0.403 and a polymer coil volume fraction of 0.277 , which is an overestimation of less than a factor of 3 . The polymer concentration at the triple point is now of the same order of magnitude as in the experiment. At the experimental colloid volume fraction $(0.444)$ theory predicts a fluid-crystal coexistence with a polymer concentration of 0.19 in the fluid, which is even closer to the experimental data.

Previously and above, we compared the free volume theory with model polymer-colloid mixtures. ${ }^{35}$ Here, we also compare the results of a protein-polysaccharide mixture of casein micelles, the main association colloids in milk, and an exocellular polysaccharide ${ }^{52}$ with the free volume theory. Casein micelles are spherical protein particles, which effectively behave as hard spheres with a number-averaged radius $R$ close to 100 $\mathrm{nm}$ in a salt solution as in milk at neutral $\mathrm{pH} .{ }^{53}$ The exocellular polysaccharide was produced by a lactic acid bacterium and has a radius of gyration of $86 \mathrm{~nm}$ and a number-averaged molar mass of $1470 \mathrm{~kg} \mathrm{~mol}^{-1} .{ }^{54} \mathrm{In}$ an aqueous solution with $0.10 \mathrm{M}$ salt this polymer behaves as in a good solvent, as can be derived from the scaling exponents from light and neutron scattering. ${ }^{54,55}$ However, it should be realized that the effective Flory-Huggins parameter $\chi$ is still close to 0.5 (0.48 for dextran) for polysaccharides in water, ${ }^{56}$ whereas $\chi$ is 0 for polymer chains in the full excluded volume limit. The phase diagram of the mixture of casein micelles and the exocellular polysaccharide (EPS) was reported in ref. 52 and the binodal points are redrawn as the filled diamonds in Fig. 6. The dashed curve is the free volume result for hard spheres mixed with ideal polymer chains for $q=0.86,{ }^{13}$ whereas the full curve is the result for polymers in the excluded volume limit mixed with hard spheres at the same size ratio. ${ }^{35}$ The theoretical critical points are given as filled circles in Fig. 6. Experimentally, the critical colloid volume fraction is expected to be located at a colloid

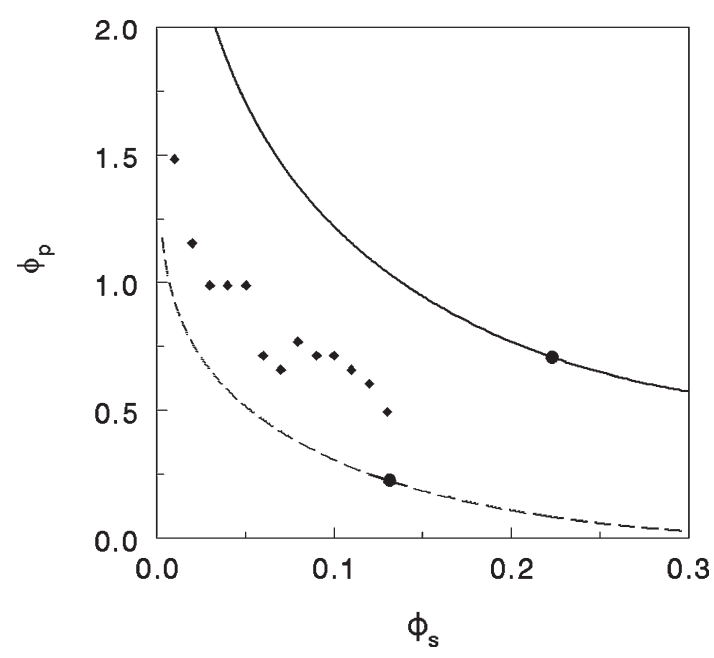

Fig. 6 Phase diagram of an exocellular polysaccharide-casein micelle mixture ${ }^{52}$ with $q=0.86$ in comparison with the free volume predictions for ideal polymer chains (dashed curves) and polymer chains in the excluded volume limit (full curves). 
volume fraction above 0.13 since at all investigated casein micelle concentrations the volume of the casein micelle rich phase was always smaller than that of the EPS-rich phase. The measured phase line lies in-between the results for fully interacting and non-interacting polymer chains. It follows that in practice many systems will phase separate in the range between the predictions for the ideal and excluded volume limit.

Finally, we compare our results with recent computer simulation results on many body polymer-colloidal sphere mixtures. $^{30,42}$ Bolhuis et al. ${ }^{30}$ made computer simulations on a mixture of colloidal spheres and Gaussian core (GC) spheres of which the interactions were mapped on self-avoiding walk computer simulations GC-GC interaction and the sphere$\mathrm{GC}$ interaction. For a size ratio $q=0.34$ the computer simulation results agree very well with our results, but both results yet hardly differ from the results for ideal polymer chains mixed with hard spheres. For $q=0.67$ and $q=1.08$ both our results, as well as the computer simulation results ${ }^{30}$ predict a significant shift in polymer coil volume fraction where phase separation occurs upward. The shift is more significant for the free volume theory. So, both approaches predict a much larger miscibility region, as compared to the ideal polymers and the predicted critical points seem to be located at a significantly larger colloid volume fraction, as compared to the results for ideal chains; a significant difference with the results for ideal polymers is that for ideal-sphere mixtures the colloid volume fraction at the critical point vanishes for large $q .{ }^{57}$ It is realized that, although the GC model has been mapped on SAW computer simulation results on the pair level, the many body GC results may differ from the exact result. It is satisfying, however, that similar trends are recovered in simulation and our free volume theory.

The reported binodals for a many-body mixture of spheres mixed with SAW polymer chains, simulated by Bolhuis et al. ${ }^{42}$ for $q=3.86,5.58$ and 7.78 are at critical colloid volume fractions of $c a .0 .24,0.25$ and 0.26 , respectively, indicating that the critical colloid volume fraction does not decrease with $q$ for large values of $q$. The minimum value for the critical volume fraction of colloidal spheres can be rationalized as follows. For ideal polymer chains the relevant polymer size increases with $q$ and so the colloid volume fraction will always decrease with $q$. In the case of interacting polymers the relevant polymer size is the correlation length. If the phase diagram shifts toward the semi-dilute region, an increase in $q$ leads to an effectively smaller correlation length, $\xi_{\mathrm{b}}$, than the radius of gyration. Deeper in the semi-dilute regime the increase in $q$ may result in such a decrease in correlation length that the effective size ratio $\xi_{\mathrm{b}} / R$ may decrease, leading to an increase in the critical colloid volume fraction. Very deep in the semi-dilute regime scaling theory indicates that only the correlation length and sphere radius are the relevant length scales, suggesting there will be a universal colloid volume fraction at the critical point (see ref. 42). In the large $q$-regime the free volume theory largely overestimates the binodal polymer coil volume fractions. As an indication, at $q=2.5$ the free volume theory [using eqn. (10)] predicts a critical point at a colloid volume fraction of 0.178 and a polymer coil volume fraction of 7.9. Even at $q=7.78$, the polymer coil volume fraction at the critical point is below $5 .^{42}$ The reason may be due to the high sensitivity of the free volume theory on the precise form of the correlation length from the RG theory. In Schäfer's book ${ }^{36}$ (page 423) the RG correlation length is compared with experimental data. It follows that the correlation length from the RG theory may predict a somewhat too small correlation length which leads to predicted binodals for $q>0.5$ that are shifted upward in the polymer concentration plane. From our theory it follows that the shift of the phase diagram toward higher polymer concentration due to interacting polymers is due to a decrease of the polymer correlation length that overwhelms the increase in the osmotic pressure of the polymer solution.

\subsection{Colloidal dispersion of platelets with added excluded volume polymer}

In order to describe the phase behavior of a mixture of platelets and polymer chains with excluded volume interaction we describe the platelets as cut spheres and use results from Zhang et al. ${ }^{17}$ For cut spheres the volume fraction $\phi_{\text {plate }}$ for platelets with a diameter $D$ and platelet thickness $L$, is defined as:

$$
\phi_{\text {plate }}=\frac{N}{V} \frac{\pi}{4}\left(L D^{2}-\frac{L^{3}}{3}\right)
$$

where $N$ is the number of platelets. Using scaled particle theory, Zhang et al. ${ }^{17}$ derived the following expression for the free volume fraction of polymer chains in a dispersion of cut spheres:

$$
\alpha=\left(1-\phi_{\text {plate }}\right) \exp \left(-\frac{A_{\mathrm{pl}} \phi_{\text {plate }}}{1-\phi_{\text {plate }}}-\frac{B_{\mathrm{pl}} \phi_{\text {plate }}^{2}}{\left(1-\phi_{\text {plate }}\right)^{2}}-C_{\mathrm{pl}} \beta P_{\mathrm{pl}} D^{3}\right),
$$

with

$$
\begin{aligned}
A_{\mathrm{pl}}= & \frac{2 \frac{\Delta}{D}\left(1+2 \frac{L}{D}-\frac{L^{2}}{D^{2}}\right)}{\frac{L}{D}-\frac{L^{3}}{3 D^{3}}} \\
& +\frac{4 \frac{\Delta^{2}}{D^{2}}\left(2 \frac{L}{D}+\left(\frac{\pi}{2}-\arcsin \left(\frac{L}{D}\right) \sqrt{\left.1-\left(\frac{L}{D}\right)^{2}\right)}\right)\right.}{\frac{L}{D}-\frac{L^{3}}{3 D^{3}}}, \\
B_{\mathrm{pl}}= & \frac{4\left(\frac{\Delta}{D}\right)^{2}\left(1+2 \frac{L}{D}-\frac{L^{2}}{D^{2}}\right)^{2}}{2\left(\frac{L}{D}-\frac{1}{3}\left(\frac{L}{D}\right)^{3}\right)^{3}}, \text { and } C_{\mathrm{pl}}=\frac{4 \pi}{3}\left(\frac{\Delta}{D}\right)^{3}
\end{aligned}
$$

The parameters $A_{\mathrm{pl}}, B_{\mathrm{pl}}$ and $C_{\mathrm{pl}}$ all depend on the depletion layer thickness $\Delta$. Since platelets are considered with $L \ll D$, the large majority of surfaces is flat for the polymer chains. This means that the depletion layer thickness near a flat plate is the physical quantity that is required, i.e. for this case we take eqn. (11) for $\Delta$, where we use the polymer density dependent correlation length from the RG theory. This means that the depletion layer thickness and hence the free volume fraction become polymer density dependent.

The only unknown in eqn. (24) is the equation of state $P_{\mathrm{pl}}$ for the cut sphere system. We use the approach of Zhang et al. ${ }^{17}$ who performed Monte Carlo simulations of cut spheres and determined the equations of state in the isotropic, nematic, and columnar phase. The simulation data were fitted to a polynomial:

$$
\beta P_{\mathrm{pl}} D^{3}=\sum_{i=1}^{K} a_{i}\left(\frac{N D^{3}}{V}\right)^{i},
$$

and a fit up to sixth order $(K=6)$, for which the fitting parameters are given in Table 1, describes the Monte Carlo data very accurately. This means that all ingredients for the free volume theory are available. We can again use the general eqn. (21) with $v_{\text {coll }}=D^{3}$ in the case of platelets and the osmotic compressibility for the solution of excluded volume chains is given by eqn. (3). The normalized free energy follows directly from eqn. (25).

In Fig. 7(a) we have depicted the experimental phase diagram, as obtained by Van der Kooij et al.. ${ }^{4}$ Their PDMSpolymer is estimated to have a radius of gyration close to 
Table 1 Transition densities and fitting parameters to the equations of state for the cut-sphere system with $L / D=0.05$, following from the simulation results of Zhang et al. ${ }^{17}$

\begin{tabular}{lclccccc}
\hline Phase & $N D^{3} / \mathrm{V}$ & $a_{1}$ & $a_{2}$ & $a_{3}$ & $a_{4}$ & $a_{5}$ \\
\hline $\mathrm{I}$ & $0-3.89689$ & 1 & 0.736951 & 0.338647 & -0.0290027 & -0.0077227 \\
$\mathrm{~N}$ & $4.1726-10.3121$ & 6.7213 & -1.16676 & 0.219941 & -0.00728876 & 0 \\
$\mathrm{C}$ & $10.9964-15.2$ & 0 & 16.504 & -4.69545 & 0.535796 & 0 \\
\hline
\end{tabular}

$33 \mathrm{~nm}$ in toluene and a molar mass of $4.2 \times 10^{5} \mathrm{~g} \mathrm{~mol}^{-1}$. This means that the overlap concentration is $4.6 \mathrm{~g} \mathrm{~L}^{-1}$. The platelets have a diameter of $208 \mathrm{~nm}$ and a thickness of $c a .14 \mathrm{~nm}$. In Figs. 7(b) and (c) phase diagrams are presented for the case
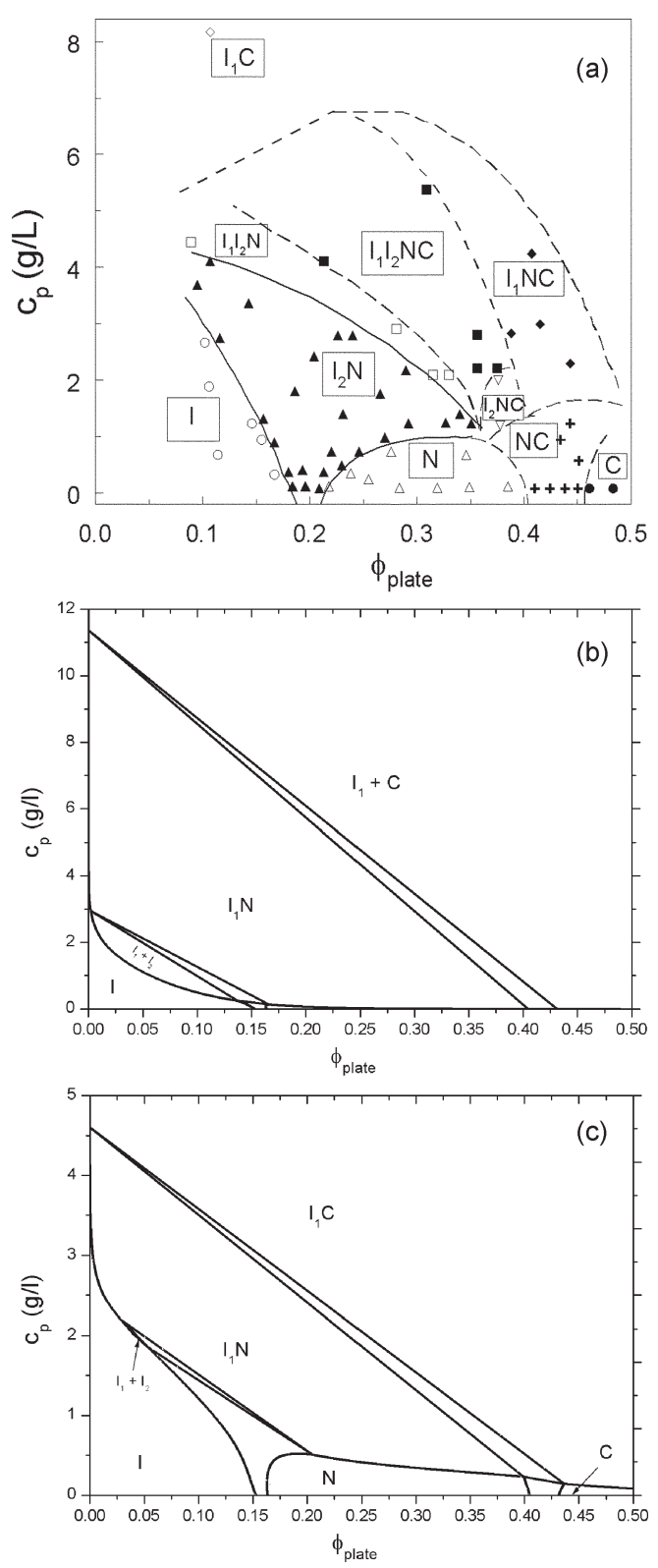

Fig. 7 (a) Redrawn experimental phase diagram from ref. 4 of a sterically stabilized gibbsite platelet/PDMS mixture with plate diameter $D=208 \mathrm{~nm}$, plate thickness $L=14 \mathrm{~nm}$ and a radius of gyration of the polymer of $33 \mathrm{~nm}$. The different phases are: $\mathrm{I}=$ fluid isotropic, $\mathrm{I}_{1}=$ isotropic gas phase, $\mathrm{I}_{2}=$ isotropic liquid phase, $\mathrm{N}=$ nematic and $\mathrm{C}=$ columnar phase. The various regions are indicated in the figure. (b) Theoretical phase diagram of a platelet/polymer mixture with $L / D=0.05$ and $R_{\mathrm{g}} / D=0.16$ for ideal polymers. Plotted is the polymer concentration (in $\mathrm{g} \mathrm{L}^{-1}$ ) versus the volume fraction of plates. (c) Phase diagram as in Fig. 7(b) but for interacting polymers.
$R_{\mathrm{g}} / D=0.16$ and $L / D=0.05$ using the theoretical description, given above. The representation in Fig. 7(c) can be directly compared to the experimental phase diagram in Fig. 7(a). The full curves are the results for platelets mixed with polymer chains in the excluded volume limit whereas the dashed curves represent the results for platelets mixed with non-interacting (ideal) polymer chains. It follows that there are significant differences between the two descriptions. First, the $I_{1}-I_{2}$ coexistence region is significantly reduced when ideal polymers are replaced with interacting polymer chains. Moreover, the extended free volume theory leads to more developed $\mathrm{I}_{1}-\mathrm{N}$ and $\mathrm{N}-\mathrm{C}$ two-phase regions compared to the those predicted from the original theory, which is in closer qualitative agreement with the experimental phase diagram in Fig. 7(a). Remarkably, no $\mathrm{I}_{1}-\mathrm{I}_{2}$ coexistence region was found experimentally. However, judging from our theoretical diagram, this region may be very small so that it will be hard to detect it in experiment. Furthermore, we can see in Fig. 7(c) that the extended theory predicts a major shift of the isotropiccolumnar phase two-phase region towards lower polymer concentrations and therefore qualitatively accounts for the experimentally observed $\mathrm{I}_{1}-\mathrm{C}$ coexistence shown in the left upper part of Fig. 7(a). Taking into account polymer excluded volume effects in the description of the phase behavior of plate-polymer mixtures thus constitutes a significant qualitative improvement over the original ideal-polymer treatment.

\section{Conclusions}

A comprehensive approach is presented for the depletion interaction and the resulting phase behavior of a colloidal dispersion with added polymer chains in the excluded volume limit. The adsorption method was used to calculate the depletion-induced interaction between colloidal particles due to non-adsorbing polymers with excluded volume interaction. Standard expressions for the correlation length and osmotic compressibility from the renormalization group theory in the excluded volume limit of polymers are used to take into account the interacting polymer solution properties. Results for the interaction are presented for the plate- and spheregeometries and are shown to agree with computer simulation results and experimental data.

As a many-body analogue of the adsorption method we use the free volume theory to describe the phase behavior of colloidal dispersions of hard particles mixed with polymer chains with excluded volume interactions. The method is versatile in the sense that the colloidal particles may have various shapes. For interacting polymers mixed with hard spheres we compare the results from our theory with a phase diagram of a biopolymer mixture. The experimental results lie in-between the two limits of ideal and full excluded volume chains. This corroborates with the fact that aqueous biopolymer solutions can be described as polymer solutions for which the polymer-solvent interaction is in-between the isothermal and theta conditions. Both for sphere-polymer and plate-polymer mixtures we conclude that incorporating excluded volume effects into the original description leads to an improved qualitative agreement with the experimental phase diagram. 


\section{Acknowledgements}

It is a pleasure to thank Ard Louis, Peter Bolhuis and Evert Jan Meijer for useful discussions and for sending us preprints and numerical data from their work. Jeroen van Duijneveldt and Shu-Dong Zhang are acknowledged for discussions on polymer-platelet mixtures and providing their data.

\section{References}

1 W. C. K. Poon, J. Phys.: Condens. Matter, 2002, 14, R859.

2 S. M. Ilett, A. Orrock, W. C. K. Poon and P. N. Pusey, Phys. Rev. E, 1995, 51, 1344.

3 J. Buitenhuis, L. N. Donselaar, P. A. Buining, A. Stroobants and H. N. W. Lekkerkerker, J. Colloid Interface Sci., 1995, 175, 46.

4 F. M. Van der Kooij, M. Vogel and H. N. W. Lekkerkerker, Phys. Rev. E., 2000, 62, 5397.

5 J.-L. Doublier, C. Garnier, C. Renard and C. Sanchez, Curr. Opin. Coll. Int. Sci., 2000, 5, 184.

6 T. Odijk, Biophys. Chem., 1998, 73, 23.

7 F. Livolant and A. Leforestier, Prog. Polym. Sci., 1996, 21, 1115.

8 S. Asakura and F. Oosawa, J. Polym. Sci., 1958, 33, 183.

9 A. Vrij, Pure Appl. Chem., 1976, 48, 471.

10 H. De Hek and A. Vrij, J. Colloid Interface Sci., 1981, 84, 409

11 A. P. Gast, C. K. Hall and W. B. Russel, J. Colloid Interface Sci., 1983, 96, 251 .

12 H. N. W. Lekkerkerker, Colloids Surf., 1990, 51, 419.

13 H. N. W. Lekkerkerker, W. C. K. Poon, P. N. Pusey, A. Stroobants and P. B. Warren, Europhys. Lett., 1992, 20, 559.

14 M. Dijkstra, J. M. Brader and R. Evans, J. Phys: Condens. Matter, 1999, 11, 10079

15 R. P. Sear and D. Frenkel, Phys. Rev. E, 1997, 55, 1677.

16 A. Stroobants and H. N. W. Lekkerkerker, Il Nuovo Cimento, 1994, 16, 949

17 S.-D. Zhang, P. A. Reynolds and J. S. Van Duijneveldt, Mol. Phys., 2002, 100, 3041; S.-D. Zhang, P. A. Reynolds and J. S. Van Duijneveldt, J. Chem. Phys., 2002, 117, 9947.

18 A. P. Chatterjee and K. S. Schweizer, J. Chem. Phys., 1998, 109, 10464; A. P. Chatterjee and K. S. Schweizer, J. Chem. Phys., 1998, 109, 10447.

19 M. Fuchs and K. S. Schweizer, Europhys. Lett., 2000, 51, 621; M. Fuchs and K. S. Schweizer, Phys. Rev. E, 2001, 64, 21514.

20 M. Fuchs and K. S. Schweizer, J. Phys.: Condens. Matter, 2002, 14, R239.

21 M. Schmidt and M. Fuchs, J. Chem. Phys., 2002, 117, 6308.

22 M. Schmidt, A. R. Denton and J. M. Brader, J. Chem. Phys., 2003, 118, 1541 .

23 A. A. Louis, P. G. Bolhuis, J. P. Hansen and E. J. Meijer, Phys. Rev. Lett., 2000, 85, 2522.

24 P. G. Bolhuis, A. A. Louis, J. P. Hansen and E. J. Meijer, J. Chem. Phys., 2001, 114, 4296.

25 P. G. Bolhuis, A. A. Louis and J. P. Hansen, Phys. Rev. E, 2001, 62, 7961 .

26 A. A. Louis, P. G. Bolhuis, E. J. Meijer and J.-P. Hansen, J. Chem. Phys., 2002, 116, 10547.

27 A. A. Louis, P. G. Bolhuis, E. J. Meijer and J.-P. Hansen, J. Chem. Phys., 2002, 117, 1893.
28 P. G. Bolhuis and A. A. Louis, Macromolecules, 2002, 35, 1860.

29 A. A. Louis, P. G. Bolhuis, R. Finken, V. Krakoviack, E. J. Meijer and J.-P. Hansen, Physica A, 2002, 306, 251.

30 P. G. Bolhuis, A. A. Louis and J.-P. Hansen, Phys. Rev. Lett. 2002, 89, 128302.

31 A. Hanke, E. Eisenriegler and S. Dietrich, Phys. Rev. E., 1999, 59, 6853.

32 F. Schlesener, A. Hanke, R. Klimpel and S. Dietrich, Phys. Rev. E., 2001, 63, 41803 .

33 R. Tuinier and H. N. W. Lekkerkerker, Eur. Phys. J. E, 2001, 6, 129. Note that in this paper we used a product function approximation. It is noted, as reported in ref. 27, that the simulation results should be adjusted slightly. Besides that an inaccurate expression for the correlation length was used. Here we use an accurate expression from ref. 36 . These shortcomings only have a small effect on the final results in ref. 33.

34 R. Tuinier, H. N. W. Lekkerkerker and D. G. A. L. Aarts, Phys Rev. E, 2002, 65, R060801.

35 D. G. A. L. Aarts, R. Tuinier and H. N. W. Lekkerkerker, J. Phys.: Condens. Matter, 2002, 14, 7551.

36 L. Schäfer, Excluded Volume Effects in Polymer Solutions, Springer Verlag, Berlin, 1999.

37 J. F. Joanny, L. Leibler and P. G. De Gennes, J. Polym. Sci. Polym. Phys. Ed., 1979, 17, 1073.

38 D. G. Hall, J. Chem. Soc., Faraday Trans., 1972, 68, 2169.

39 Y. Mao, P. Bladon, H. N. W. Lekkerkerker and M. E. Cates, Mol. Phys., 1997, 92, 151.

40 R. Tuinier and H. N. W. Lekkerkerker, Macromolecules, 2002, 35 3312 .

41 G. A. Vliegenthart and P. Van der Schoot, Europhys. Lett., 2003, 62, 600 .

42 P. G. Bolhuis, A. A. Louis and E. J. Meijer, Phys. Rev. Lett., 2003, 90, 68304

43 R. Tuinier, Eur. Phys. J. E, 2003, 10, 123.

44 R. Verma, J. C. Crocker, T. C. Lubensky and A. G. Yodh, Macromolecules, 2000, 33, 177.

45 A. Milling and S. Biggs, J. Colloid Interface Sci., 1995, 170, 604

46 J. Y. Walz, Curr. Opin. Coll. Int. Sci., 1997, 2, 600.

47 C. Bechinger, D. Rudhardt, P. Leiderer, R. Roth and S. Dietrich, Phys. Rev. Lett., 1999, 83, 3960.

48 Y. N. Ohshima, H. Sakagami, K. Okumoto, A. Tokoyoda, T. Igarashi, K. B. Shintaku, S. Toride, H. Sekino, K. Kabuto and I. Nishio, Phys. Rev. Lett., 1997, 78, 3963.

49 H. Reiss, H. L. Frisch and J. L. Lebowitz, J. Chem. Phys., 1959, 31, 369.

50 J. L. Lebowitz, E. Helfand and E. Praestgaard, J. Chem. Phys, $1965, \mathbf{4 3}, 774$

51 A. Moussaïd, W. C. K. Poon, P. N. Pusey and M. F. Soliva, Phys. Rev. Lett., 1999, 82, 225.

52 R. Tuinier and C. G. de Kruif, J. Chem. Phys., 1999, 110, 9296.

53 C. G. de Kruif, J. Dairy Sci., 1998, 81, 3019.

54 R. Tuinier, P. Zoon, C. Olieman, M. A. Cohen Stuart, G. J. Fleer and C. G. de Kruif, Biopolymers, 1999, 49, 1.

55 R. Tuinier, C. Holt, P. A. Timmins and C. G. de Kruif, J. Appl. Crystallogr., 2000, 33, 540.

56 M. M. G. Koning, J. van Eendenburg, D. W. de Bruijne, in Food Colloids and Polymers, ed. E. Dickinson and P. Walstra, Royal Soc. Chem., Cambridge, 1993, p.103.

57 R. P. Sear, Phys. Rev. Lett., 2001, 86, 4696. 